ARTIGO

\title{
ETIQUETAS INTELIGENTES NA ADMINISTRAÇÃO PÚBLICA: ANÁLISE DA VIABILIDADE NO CONTROLE PATRIMONIAL DA UNIVASF ${ }^{1}$
}

\author{
Cátia Valéria dos Santos Passos Brito ${ }^{2}$ \\ Welson Barbosa dos Santos ${ }^{3}$ \\ Cristiane Xavier Galhardo ${ }^{4}$ \\ Vivianni Marques Leite dos Santos 5
}

\begin{abstract}
RESUMO
A realização de um inventário não é uma tarefa fácil, principalmente em universidades federais, que possuem muitos bens permanentes. A gestão patrimonial é realizada através de procedimentos de identificação, tombamento, localização, contagem, preservação e desfazimento de bens. Devido a grande quantidade de informações e necessidade de segurança, confiabilidade e celeridade nos processos, deve-se buscar alternativas para melhorar a gestão, reduzir índices de não conformidades e aprimorar o planejamento de compras. O sistema RFID (Radio-Frequency Identification), por exemplo, funciona por meio da comunicação via radiofrequência, com uso de um chip passivo inserido em etiqueta inteligente. Dessa forma, o objetivo deste artigo é analisar a viabilidade do uso da tecnologia RFID para o aperfeiçoamento da gestão patrimonial na Universidade Federal do Vale do São Francisco - Univasf por meio do levantamento de suas vantagens, desvantagens e custos para implantação. Para isto, são realizadas pesquisas em periódicos e bases de patentes, além da realização de entrevistas e análise documental. Entre os principais resultados, constata-se que o processo de gerenciamento de bens da Univasf é realizado de forma manual e desgastante para servidores membros de comissão, para a qual são convocados sem consulta prévia, verificando-se ainda que os prazos são, comumente, ultrapassados. Por outro lado, o custo para implantação do sistema RFID para controle do número atual de bens da Univasf corresponde a apenas $0,78 \%$ do valor alocado para as ações orçamentárias referentes às despesas correntes e investimentos, constituindo fatores favoráveis a sua aquisição pela Instituição.
\end{abstract}

Palavras-chave: Radiofrequência. Gestão patrimonial. Universidades públicas. Internet das coisas.

\footnotetext{
${ }^{1}$ Como citar este artigo: BRITO, Cátia Valéria dos Santos Passos; SANTOS, Welson Barbosa dos; GALHARDO, Cristiane Xavier; SANTOS, Vivianni, Marques Leite dos. Etiquetas inteligentes na administração pública: análise da viabilidade no controle patrimonial da UNIVASF. ForScience: revista científica do IFMG, Formiga, v. 7, n. 2, e00661, jul./dez. 2019. DOI: 10.29069/forscience.2019v7n2.e661.
}

\footnotetext{
${ }^{2}$ Autor para correspondência: Cátia Valéria dos Santos Passos Brito, e-mail: catia.valeria@univasf.edu.br

${ }^{3}$ Autor para correspondência: Welson Barbosa dos Santos, e-mail: welson.santos@univasf.edu.br

${ }^{4}$ Autor para correspondência: Cristiane Xavier Galhardo, e-mail: cristiane.galhardo@univasf.edu.br

${ }^{5}$ Autor para correspondência: Vivianni Marques Leite dos Santos, e-mail: vivianni.santos@univasf.edu.br
} 


\section{INTRODUÇÃO}

A tecnologia por Radiofrequência (RFID) é baseada na utilização de ondas eletromagnéticas (de radiofrequência), que permitem transmitir dados de identificação ou localização de produtos, como: componentes, caixas, veículos, ativos e máquinas (MOTA, 2012). De acordo com Santini (2008), devido a possibilidade de ser usada em várias áreas, essa tecnologia pode ser considerada como uma aliada à melhoria de quaisquer processos de gestão.

Sabe-se que tal tecnologia não é recente, tendo origem através da criação de sistemas de radares que foram utilizados na Segunda Guerra Mundial (USTUNDAG; TANYAS, 2009, LANDT, 2005; RODRIGUEZ et al., 2012). Esses sistemas foram criados pelo físico escocês Robert Alexander Watson-Watt com o objetivo de alertar a aproximação de aviões inimigos (MOTA, 2012). A aplicação em empresas brasileiras ocorreu somente após o ano 2000, quando houve redução significativa nos custos para sua utilização.

O uso de tecnologia por radiofrequência pode constituir alternativa para melhorias dos processos em ambientes organizacionais, dado que permite o acesso as informações sobre um determinado produto, apenas por meio de um chip inserido em etiquetas. Tratam-se de aplicações resultantes da comunicação objeto-objetivo e objeto-humano, conhecida como Internet das Coisas (Internet of Things - IoT).

O controle patrimonial, nas Instituições Públicas, por exemplo, refere-se ao gerenciamento de todos os bens móveis e imóveis, e esse gerenciamento requer bastante esforço e confiabilidade para garantir registro de informações corretas, conforme a Instrução Normativa SEDAP nº 205, 08 de abril de 1988, tais como a localização física exata dos bens, com detalhes desde sua aquisição, estado de conservação até, se for o caso, o seu desfazimento (BERNARDES, 2009), de modo que o uso de etiquetas pode agilizar o processo e dar maior segurança.

Outros aspectos relevantes para inovação em controle patrimonial estão no caráter obrigatório da realização do inventário nas instituições públicas, conforme a Lei $\mathrm{n}^{\circ} 4.320$, de 1964, e na necessidade da eficiência derivada da economicidade na Administração, destacada por Albiero e Silva (2018), o que significa o ato de gerar economia proporcionada pela otimização da relação custo-benefício.

A Universidade Federal do Vale do São Francisco - Univasf é multicampi, com estrutura organizacional composta por 06 campi em três diferentes estados do Nordeste do Brasil. De acordo com informações obtidas junto à Pró-Reitoria de Planejamento e 
Desenvolvimento Institucional (UNIVASF, 2018), o inventário é anual, realizado ao final de cada exercício e o crescente número de bens permanentes adquiridos ao longo dos anos, somam mais de sessenta (60) mil itens. Todas as informações são obtidas manualmente, apresentadas em planilha eletrônica, com informações já pré-informadas de tombo de todo o acervo.

Mesmo existindo o zelo, dedicação e eficiência por parte dos servidores que atuam realizando o controle patrimonial, observa-se sobrecarga de trabalho devido ao dimensionamento inadequado dos recursos humanos, aos procedimentos realizados por falta de padronização e, ainda, a não utilização de tecnologias da informação, acabam prejudicando a realização de um controle patrimonial eficaz. Narciso (2008) também relatou desafios na Embrapa Informática Agropecuária semelhante àqueles constatados na Univasf.

Visando contribuir para redução dos desafios supracitados e promover maior celeridade e confiabilidade ao processo de inventário, o objetivo desse trabalho é realizar um estudo sobre a viabilidade para implementar a tecnologia de identificação por RFID no processo de gestão patrimonial na Univasf. Espera-se que os resultados obtidos contribuam para a melhor tomada de decisão quanto a sua implementação na Instituição, bem como sirva de referência para sua aplicação em outros órgãos públicos ou privados.

\section{METODOLOGIA}

O delineamento do estudo tem natureza aplicada, uma vez que objetiva gerar conhecimentos para aplicação em curto prazo. Para isto, foram utilizadas pesquisas bibliográficas e de desenvolvimento tecnológico, bem como análise documental, em uma abordagem qualitativa com a finalidade de analisar o potencial do uso da tecnologia de identificação por radiofrequência (RFID).

Trata-se de um estudo de caso que é um método recomendável na exploração de novos processos ou descobertas, bem como na verificação da aplicabilidade na prática dos elementos de uma teoria. Caracteriza-se por ser um estudo adequado por exigir uma descrição ampla e profunda de um determinado fenômeno organizacional (YIN, 2015).

Quanto aos objetivos e abordagem, a pesquisa é classificada como qualitativoexploratória. A análise científica inicial foi direcionada aos artigos científicos publicados, que visavam analisar o uso das etiquetas RFID. Os bancos de dados de periódicos eletrônicos pesquisados foram: Scielo, Google Acadêmico e Periódicos Capes. 
A utilização da abordagem exploratória serviu para melhor entendimento quanto ao processo de controle de patrimônio em tempo real, por meio da inovação tecnológica, com o objetivo de se aprofundar no assunto e tornar mais fácil a aplicabilidade do que está sendo proposto.

A busca patentária foi realizada por meio do site de acesso público, chamado base nacional do Instituto Nacional da Propriedade Industrial - INPI. Este site é uma ferramenta oficial que possui uma biblioteca especializada em Propriedade Intelectual, Inovação e Tecnologia em geral. Para a referida busca foi utilizada a palavra-chave "RFID”, na descrição dos campos "título" e "resumo".

Após a busca, os dados foram tratados por meio de análise quanto às áreas tecnológicas, organizados com o auxílio de recursos como tabelas e figuras para facilitar a compreensão do tema proposto e compilados para geração de conhecimento.

Como parte da coleta para o diagnóstico dos procedimentos atuais, foram realizadas entrevistas semiestruturadas com o Pró-Reitor de Planejamento e Desenvolvimento Institucional e o Diretor de Planejamento da Univasf. Em paralelo, foi feita análise documental através de materiais oficiais (planilhas e relatórios), ampliando o resultado da análise (GIL, 2008).

Por fim, para análise do custo para implantação do sistema RFID na Univasf, foram solicitados orçamentos para implantação do referido sistema a partir de 02 empresas e realizada a comparação do valor aproximado de aquisição com o valor alocado para as Ações Orçamentárias referentes as despesas correntes e investimentos da Instituição. Devido ausência do registro de tempo dedicado pela comissão designada para fazer os inventários, não é possível mensurar os gastos anuais para a atendimento a essa demanda.

\section{DESENVOLVIMENTO}

Apesar de constituir uma tecnologia bastante antiga, as evidências de utilização de RFIDs ainda estão apenas em grandes empresas. Na década de 60, por exemplo, já eram utilizados em grandes lojas de departamentos e em bibliotecas, para proteção de inventários, aeroportos, etc. (QUADRO 1).

Na década seguinte (1970-1979), os Estados Unidos foram pioneiros na proteção de propriedade intelectual a partir do uso de RFID para desenvolvimento de sistema para rastreamento de material radioativo e gado (QUADRO 1). 
BRITO, Cátia Valéria dos Santos Passos; SANTOS, Welson Barbosa dos; GALHARDO, Cristiane Xavier; 5 SANTOS, Vivianni, Marques Leite dos. Etiquetas inteligentes na administração pública: análise da viabilidade no controle patrimonial da UNIVASF

\begin{tabular}{|c|l|}
\hline PERÍODO & \multicolumn{1}{c|}{ EVENTOS } \\
\hline 1940 a 1950 & Primeira utilização da tecnologia, durante segunda Guerra Mundial. \\
\hline 1950 a 1960 & $\begin{array}{l}\text { Cientistas dos Estados Unidos, Europa e Japão apresentaram pesquisas sobre a utilização } \\
\text { de RFID para identificação remota de objetos. }\end{array}$ \\
\hline 1960 a 1970 & $\begin{array}{l}\text { Empresas introduziram novos usos de RFID em aplicações menos complexas e mais } \\
\text { amplamente usadas como, por exemplo, proteger os itens de inventário, tais como } \\
\text { vestuário em lojas de departamento e livros em bibliotecas. }\end{array}$ \\
\hline 1970 a 1980 & $\begin{array}{l}\text { Primeira patente americana para um sistema RFID ativo com memória regravável e } \\
\text { patente para um sistema passivo. Desenvolvimento de sistema para rastreamento de } \\
\text { material radioativo e gado. Início da utilização em HF (High Frequency). }\end{array}$ \\
\hline 1990 a 2000 & $\begin{array}{l}\text { Alguns estados americanos adotaram um sistema de gerenciamento de tráfego baseada no } \\
\text { uso de leitores que poderia detectar etiquetas de protocolo. Utilização da tecnologia na } \\
\text { Europa em pedágios, ferrovias e controle de acesso. Aplicações de pedágios e ferrovias } \\
\text { apareceram em outros continentes. Desenvolvimento de circuitos integrados e melhorias } \\
\text { com UHF possibilitando aplicações na cadeia de suprimentos. A tecnologia começa a fazer } \\
\text { parte da vida das pessoas. }\end{array}$ \\
\hline Depois de 2000 & $\begin{array}{l}\text { Exigência por parte do Departamento de Defesa dos EUA e Walmart, da adoção da } \\
\text { tecnologia pelos seus fornecedores. Redução dos custos da adoção da tecnologia. } \\
\text { Utilização da tecnologia no Brasil em algumas empresas, por exemplo HP, Ceitec e } \\
\text { Unilever. }\end{array}$ \\
\hline
\end{tabular}

Quadro 1 - Resumo do histórico do desenvolvimento do RFID

Fonte: Adaptado de Landt (2005) e Rodriguez et al. (2012)

Apenas a partir do ano de 2000, em algumas empresas brasileiras, houve redução significativa nos custos da utilização da tecnologia RFID, como por exemplo, a Unilever, Grupo Pão de Açúcar, Procter\&Gamble, Gillete, CHEP e Accenture (QUADRO 1). Cinco anos depois, o Wal-Mart já havia instalado o sistemas RFID em seus 100 maiores distribuidores de produtos, resolvendo grande parte de seus problemas de estoque (IDTECHEX, 2005).

\subsection{Funcionamento da tecnologia RFID}

O sistema RFID (Radio Frequency IDentification), através de ondas de rádio, permite a transmissão da identidade de objetos ou pessoas. Esse sistema pode ser enquadrado na categoria de tecnologia de identificação automática (BERTAGLIA, 2013). Com essa tecnologia existem vantagens no uso em relação ao código de barras, que é a possibilidade de ser lida sem contato visual. Mesmo que os bens estejam embalados, dentro de caixas ou ainda encostados na parede e de difícil acesso, as etiquetas RFID poderão ser lidas e detectadas.

A tecnologia RFID é composta de uma etiqueta inteligente (tag), contendo um microchip onde armazena os dados de identificação dos bens. A antena permite que o chip transmita a informação de identificação a um leitor. Os leitores, por sua vez, captam a onda de radiofrequência através de uma antena que é conectada a um software no computador, e todas 
e todas as informações são gerenciadas pelo sistema RFID (ROUSSOS, 2006) (FIG. 1).

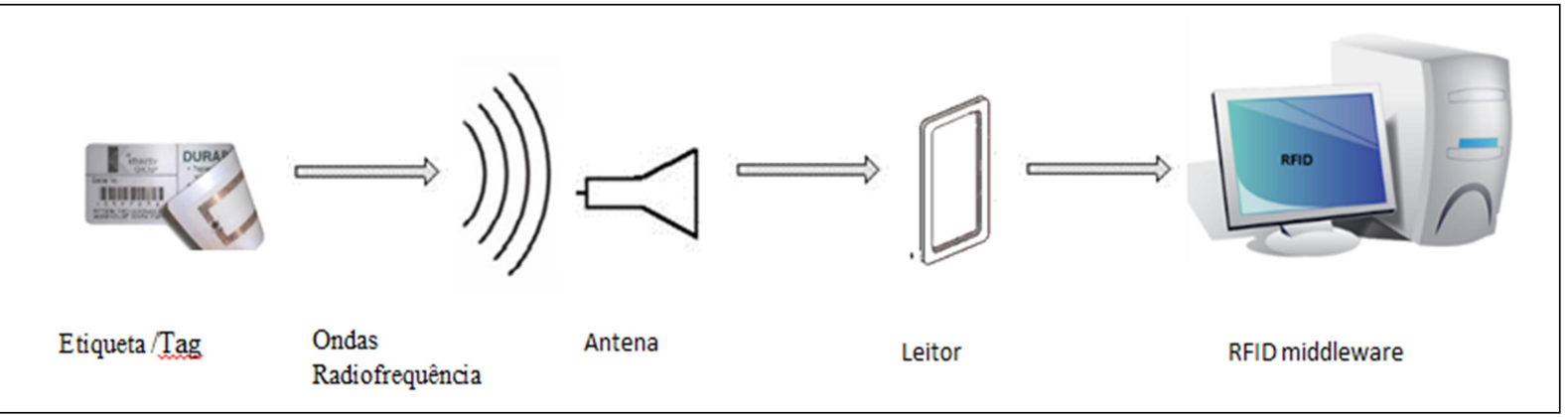

Figura 1 - Funcionamento do sistema RFID

Fonte: Elaborada pelos autores a partir de Roussos (2006).

As tags tem a capacidade de armazenamento de informações muito maior que os códigos de barras, além de conseguirem transmitir, a partir da etiqueta, dados como a identificação ou a localização de um "bem”. Além de outras informações como o preço, cor, data de compra, etc. (PREDIGER; SILVEIRA; FREITAS, 2016).

O principal componente de uma etiqueta RFID é o chip, devido conter a memória onde são armazenados os dados. Funciona enviando dados ao leitor através de ondas de rádio, transmitindo a identificação do "bem" a partir dos dados contidos na etiqueta (MOROZ, 2004).

De acordo com Costa (2018), existem dois tipos principais de etiquetas ou tags RFID: as passivas e as ativas. As tags passivas são alimentadas pela energia das ondas eletromagnéticas emitidas pelo leitor, funcionando apenas quando entram no campo de leitura do mesmo. As tags ativas possuem pequenas baterias embutidas em seus circuitos como fonte de energia, isso permite que sejam detectadas de distâncias maiores, porém são mais caras e maiores do que as etiquetas passivas (QUADRO 2).

\begin{tabular}{|l|l|l|}
\hline CARACTERÍSTICAS & \multicolumn{1}{|c|}{ PASSIVA } & \multicolumn{1}{c|}{ ATIVA } \\
\hline Distância & Até $10 \mathrm{~m}$ & Até $50 \mathrm{~m}$ \\
\hline Fonte de Energia & Transmitida pelo leitor & Bateria interna ou transmitida pelo leitor \\
\hline Multi leitura & 3000 etiquetas & 1000 etiquetas \\
\hline Sensor (temp., etc.) & Não & Sim \\
\hline Vida útil & Ilimitada & 3 a 5 anos (limitada pela bateria) \\
\hline Custo & Baixo & Alto \\
\hline Tamanho & Pequena & Grande \\
\hline Exemplos & $\begin{array}{l}\text { Identificação, rastreamento e } \\
\text { localização }\end{array}$ & Logística (container) \\
\hline
\end{tabular}

Quadro 2 - Características das etiquetas/tags RFID

Fonte: Adaptado de Oda (2014).

ForSci.: r. cient. IFMG, Formiga, v. 7, n. 2, e00661, jul./dez. 2019. 
Uma tag RFID ativa é mais cara que uma tag passiva. Sendo assim, para ficar viável a instalação de um sistema RFID quando o número de etiquetas a ser utilizado é grande, o ideal é usar tag RFID passivas, porque tem custo bem menor. Ressalta-se que, essas tags têm alcance menor do que as tags ativas, porém possuem a vantagem de, além de mais baratas, não precisarem de bateria e assim a etiqueta vai sempre funcionar, não precisando troca de baterias (NARCISO, 2008).

Para Sanghera (2007), o leitor é um dispositivo com a função de comunicar-se com as tags RFID. Possui uma ou mais antenas, que são responsáveis pela emissão das ondas de rádio e recebimento dos sinais de volta da etiqueta. Para produzir dados é necessário que todas as captações de dados estejam conectadas a um computador, onde um software fará o registro e controle relativo à operação dos leitores. Esses softwares são responsáveis por todo o controle do sistema RFID, gerenciando os dados capturados pelos leitores e integrando o fluxo de dados entre as tags e o sistema que solicita as informações desta tag (PREDIGER; SILVEIRA; FREITAS, 2016).

\subsection{Aplicação da tecnologia RFID no Brasil}

No Brasil, o primeiro depósito de patente que tem alguma relação com RFID ocorreu em 1996 (GRÁFICO 1), onde a invenção proporciona um método e um aparelho que aumenta a probabilidade de identificação de uma série de transmissores que estão todos transmitindo dados para um receptor. Entretanto, somente a partir de 2005, o crescimento foi mais expressivo, atingindo a quantidade máxima de pedidos no ano de 2014 (52). O baixo número de depósitos no ano de 2017 não permite concluir que houve redução, uma vez que é assegurado o período de sigilo de 18 meses, sendo identificado um total de 544 pedidos de patentes publicados nessa temática (GRÁFICO 1). 


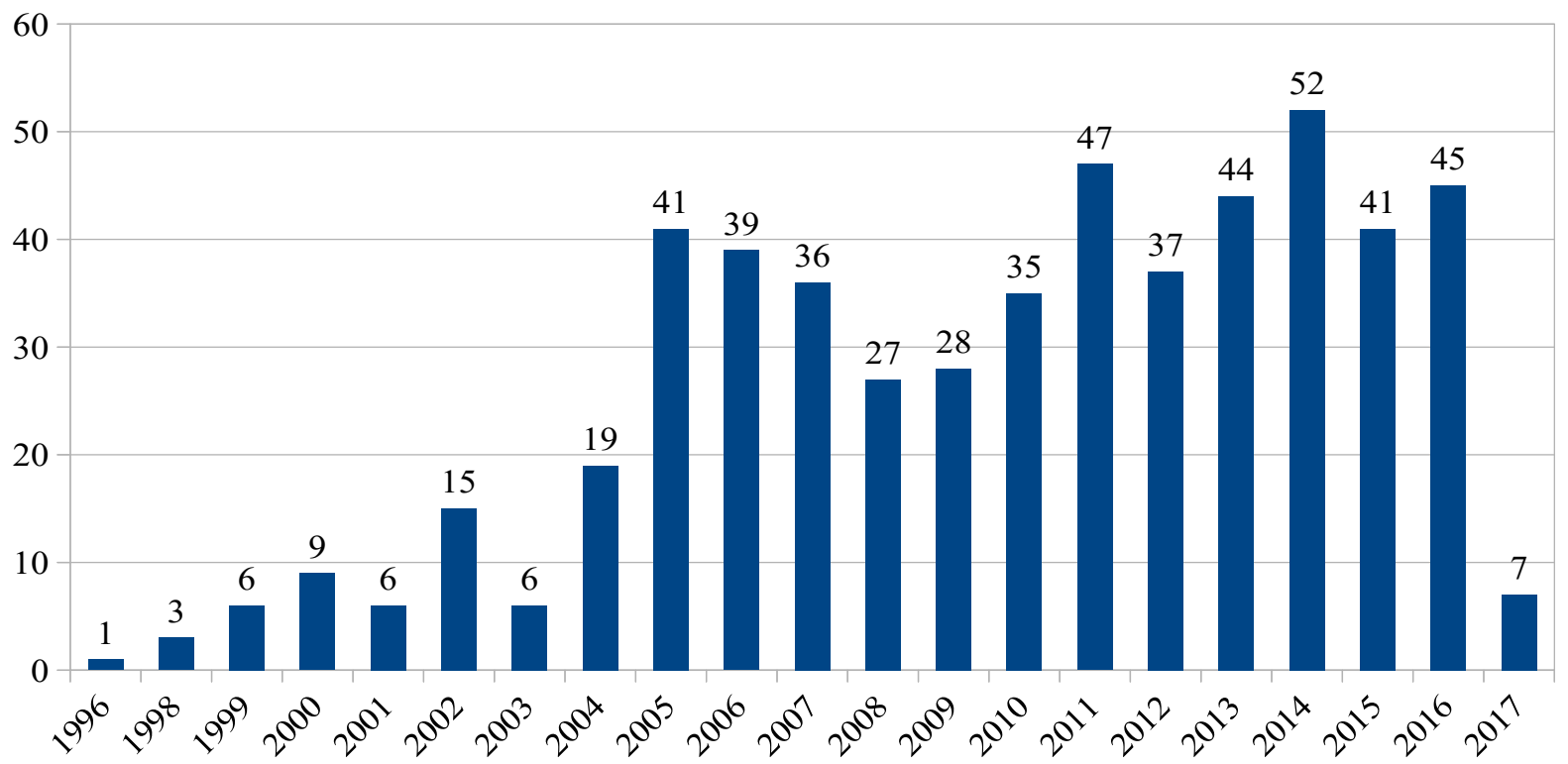

Gráfico 1 - Pedidos de depósitos com a palavra RFID na descrição do título e resumo Fonte: INPI (2018).

Ao analisar os pedidos de depósito de patentes por Classificação Internacional de Patentes (IPC - International Patents Classification), conforme Acordo de Estrasburgo (1971), constatou-se que a maioria $(65,4 \%)$ está na seção G (Física) (Tabela 1), subseção Instrumentos, com 356 ocorrências. Em segundo lugar vem os pedidos do campo tecnológico H (Eletricidade) com 83 ocorrências, e em seguida os da seção B (Operações de processamento/transporte) com 46 depósitos (TAB. 1 e 2).

Dado que na subseção Instrumentos, encontram-se pedidos relacionados a identificação, apresentação, transportes e manipulação de dados; mecanismos de contagem de objetos; processamento de dados de imagem ou geração, entre outros, a análise de viabilidade de aplicação proposta neste estudo enquadra-se nesta classificação.

Tabela 1 - Pedidos de patentes por classificação IPC.

\begin{tabular}{lcc}
\hline Campo tecnológico & Quantidade & Percentual \\
\hline A- Necessidades humanas & 30 & 5,5 \\
B- Operações de processamento/transporte & 46 & 8,5 \\
C- Química/metalurgia & 4 & 0,7 \\
D-Têxteis/papel & 6 & 1,1 \\
E- Construções fixas & 13 & 2,4 \\
F- Eng. mecânica/iluminação/aquecimento/armas/explosão & 5 & 0,9 \\
G- Física & 356 & 65,4 \\
H- Eletricidade & 83 & 15,3 \\
Não informado & 1 & 0,2 \\
\hline
\end{tabular}

Fonte: INPI (2018). 
Tabela 2 - Pedidos de patentes por subseção do IPC.

\begin{tabular}{llc}
\hline Seção & Subseção & Quantidade \\
\hline A & Agricultura & 6 \\
A & Artigos pessoais ou domésticos & 4 \\
A & Produtos alimentícios; tabaco & 5 \\
A & Saúde; salvamento; recreação & 15 \\
B & Conformação & 8 \\
B & Impressão & 3 \\
B & Separação; mistura & 4 \\
B & Transporte & 31 \\
C & Metalurgia & 1 \\
C & Química & 3 \\
D & Têxteis ou materiais flexíveis não incluídos em outro local & 6 \\
E & Edificação & 1 \\
E & Perfuração do solo; mineração & 12 \\
F & Armas; explosão & 2 \\
F & Engenharia em geral & 3 \\
G & Instrumentos & 356 \\
H & Eletricidade & 83 \\
- & Não informado & 1 \\
\hline Fonte: INPI (2018). &
\end{tabular}

\subsection{Diagnóstico quanto aos procedimentos adotados na Univasf até 2018}

De acordo com a Instrução Normativa SEDAP nº 205/88, o inventário físico é o meio de controle para a verificação dos equipamentos e materiais permanentes, em uso no órgão ou entidade. No âmbito da Universidade Federal do Vale do São Francisco - Univasf, o gerenciamento do ativo patrimonial é competência do setor de Patrimônio, hierarquicamente ligado a Pró-Reitoria de Planejamento, Administração e Desenvolvimento Institucional PROPLADI (UNIVASF, 2018).

Para a realização do inventário, realizado anualmente, é necessária a cooperação entre os diversos agentes envolvidos com o controle patrimonial. Esses agentes são: o Reitor, a Comissão Inventariante, o Setor de Administração de Materiais Permanentes - SAMP e os responsáveis por guarda de bens (UNIVASF, 2018). Após o Reitor designar a Comissão de Inventário, são iniciados os procedimentos operacionais padrões de contagem e conferência dos bens.

A comissão designada gerencia a distribuição de uma lista física contendo dados dos itens, sua respectiva localização e responsabilidade organizacional. A partir dessa lista, é feita a localização dos bens tombados em cada ambiente manualmente, ou seja, verificando um 
bem de cada vez. Os usuários dos itens sob sua guarda, verificam o relatório e atualizam os dados registrados conforme a situação existente no local.

Todas as informações são prestadas em planilha de Excel, com informações já préinformadas de tombo de todo o acervo, e em seguida, devolvidas pelo servidor para registro e segurança da informação. Para os itens não localizados, é necessário procurar novamente o item naquela sala onde o mesmo está localizado; certificar-se de que o item não foi encontrado em outra sala; entrar em contato com o responsável pelo bem e questionar sobre a presença física do patrimônio; informar, ao final do trabalho, através de e-mail específico, os itens que mesmo após os procedimentos descritos, não foram localizados (SILVA, 2018).

Finalizado em cada campus, a Comissão encaminha ao SAMP a planilha de bens localizados, bem como as Declarações de bens de terceiros utilizados na Univasf. Em seguida, estas informações são atualizadas no sistema de patrimônio. Posteriormente, após realizar os lançamentos de todos os locais de guarda, o SAMP emite para a Comissão de Inventário os seguintes relatórios: relação de bens não localizados e bens localizados; por estado de conservação e por localização e número patrimonial. Na conclusão do inventário, a Comissão elabora o relatório final e encaminha ao Gestor da Unidade.

Portanto, a realização do trabalho da Comissão de Inventário da Univasf é manual e desgastante. $\mathrm{O}$ volume de itens a serem inventariados é grande e a necessidade de tomar nota, um a um, gera um enorme esforço humano, aumentando consideravelmente, a possibilidade de erros. Tal dificuldade implica, inclusive, na baixa motivação dos membros inventariantes que são convocados a fazer parte da Comissão, pois essa indicação dos membros é feita sem consulta prévia (ANDRADE, 2018).

O procedimento de inventário tem prazo para ser executado até dia 31 de dezembro do ano vigente, porém na Univasf, esse prazo é ultrapassado, sempre é necessária a concessão de um período superior para realização dos levantamentos, visando à execução de um trabalho qualitativo (ANDRADE, 2018).

Ao levar em consideração a metodologia de inventário adotada hoje, pela Instituição, onde há demandas por recursos materiais, veículos, diárias e passagens, e ainda, a designação de aproximadamente 35 servidores temporariamente, para comporem a comissão executora dos trabalhos, pode-se constatar que o custo benefício na adoção da nova tecnologia é vantajoso.

\subsection{Custo para implantação da tecnologia RFID}


Para análise dos custos foram considerados suprimentos, materiais permanentes, incluindo coletores de dados e respectivo software, etiquetas metálicas, conforme orçamento de itens básicos da estrutura de funcionamento do RFID, sem levar em consideração a preparação da base contábil, conciliação físico x contábil e ajustes das sobras (QUADRO 3).

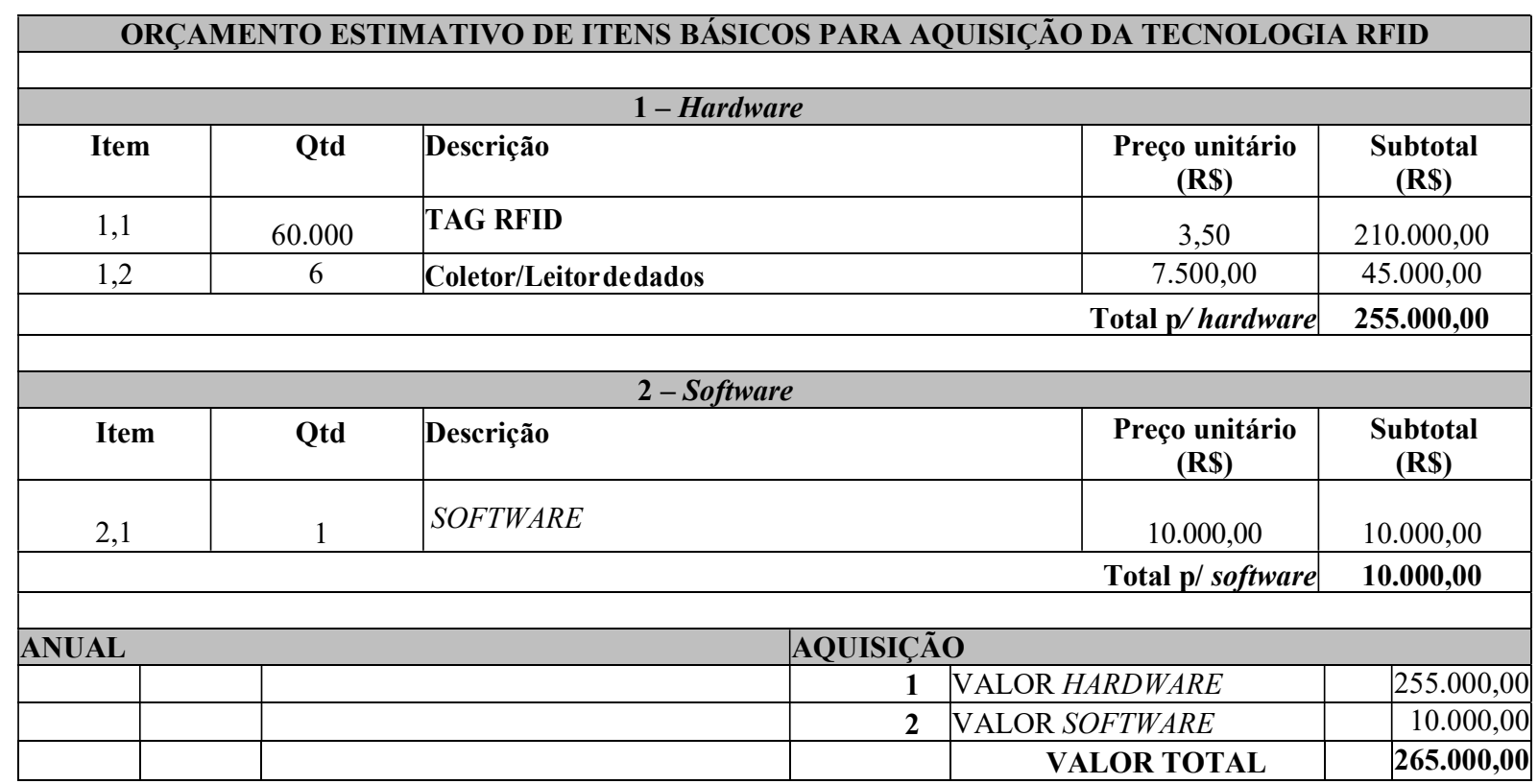

Quadro 3 - Média de orçamento - Controle patrimônio com uso de RFID

Fonte: Elaborado pelos autores.

De acordo com o orçamento apresentado para a solução de Gestão de Bens Patrimoniais, baseada na tecnologia RFID, para um quantitativo estimado de 60 mil itens, será necessário um investimento de aproximadamente duzentos e sessenta e cinco mil reais $(\mathrm{R} \$ 265.000,00)$. Esse valor corresponde a cerca de $0,78 \%$ do valor alocado $\left(\mathrm{R} \$ 33.623 .935,00^{6}\right)$ para as Ações Orçamentárias referentes às despesas correntes mais os investimentos da Univasf no ano de 2018 (UNIVASF, 2018). Ademais, não há possibilidade de mensurar os gastos com a metodologia tradicional empregada para realização do inventário, dado que verificou-se que não são feitos quaisquer registros acerca do tempo dedicado por cada servidor designado para aquela atividade.

Apesar de não poder mensurar, quantitativamente, os ganhos financeiros com a adesão a essa tecnologia, as evidências para conclusão sobre sua viabilidade estão também nas suas

\footnotetext{
6 A partir desse valor, são custeadas despesas como: contas de água, energia, telefonia e internet; serviços terceirizados (vigilância, motoristas, apoio administrativo, limpeza); combustível e manutenção de veículos; manutenção predial; passagens e diárias; publicações; aquisição de materiais de consumo (água, café, açúcar, gás, material de expediente, serviços gráficos, etc.) e despesas LEDS. Bem como são contempladas: obras; aquisição de equipamentos diversos de uso comum; mobiliários; veículos e despesas LEDS.
} 
diversas vantagens, com destaque para a maior segurança e agilidade no processo, o qual requer esforços adicionais as atribuições inerentes aos servidores.

\subsection{Vantagens e Desvantagens do uso da tecnologia RFID}

Para decidir pela implementação de ações em uma organização por meio do uso de novas tecnologias, faz-se necessário identificar as vantagens e desvantagens inerentes aquelas tecnologias, de modo a constituir requisitos para tomada de decisão quanto a viabilidade da implementação. No caso da RFID, Hodges e Mcfarlane (2005) registraram diversas vantagens:

a) Os sistemas com RFID tem maior confiabilidade, são capazes de funcionar em diversos tipos de ambientes;

b) Tem maior durabilidade, faz leitura automática e simultânea;

c) A RFID reduz ou elimina erros humanos (aleatórios);

d) As informações são enviadas, recebidas e processadas rapidamente;

e) Tem facilidade de leitura, não há necessidade do contato visual nem físico entre o produto e o leitor;

f) Capacidade de identificar vários objetos com etiquetas em um mesmo instante;

g) Rastreabilidade: capacidade de rastreamento de produtos e seres vivos;

h) Maior capacidade de armazenamento de dados, informações podendo ser escritas/reescritas e apagadas.

Por outro lado, o mesmo autor relatou as seguintes desvantagens:

a) Custo de um sistema RFID é alto, em comparação com outras tecnologias similares, como o código de barras;

b) Dificuldade de padronização das frequências utilizadas para que os produtos sejam lidos de maneira uniforme;

c) Determinados ambientes com materiais metálicos podem causar uma interferência no sistema e causar problemas em seu desempenho, necessitando escolha adequada do tipo de etiqueta. 
Ademais, a análise permite concluir que as vantagens oportunizadas pelo uso de RFID geram ganhos significativos para celeridade do processo de controle patrimonial, cujas desvantagens não inviabilizam sua adoção, desde que adequadamente consideradas durante processo de tomada de decisão, conforme descrito nos próximos itens.

\section{CONCLUSÃO}

O trabalho contém apresentação da tecnologia RFID de forma geral e destaca sua aplicação na área do controle patrimonial, com capacidade de rastrear ativos e pessoas. Seu diferencial de precisão e confiabilidade é muito importante para realização de inventários. A principal vantagem de sua aplicação em lugar do modelo manual tradicional, está na possibilidade de monitorar os bens públicos, de maneira célere e eficaz, proporcionando maior segurança na rastreabilidade do patrimônio.

Entre os principais resultados, constatou-se que o processo de gerenciamento de bens realizado de forma manual é desgastante e de baixa confiabilidade, dado o acúmulo de tarefas e erros aleatórios advindos de observações humanas, verificando-se ainda que os prazos são comumente ultrapassados.

A principal contribuição desse trabalho foi demonstrar que, usando etiquetas inteligentes, os gestores poderão ter mais agilidade na localização, distribuição e rastreamento dos bens, bem como, o controle de movimentação dos mesmos, com possibilidade de resolução de muitos problemas de forma automática e eficiente.

A principal desvantagem está relacionada ao possível alto custo inicial $(\mathrm{R} \$ 265.000,00)$ para o cadastro e controle de 60 mil itens. Entretanto, deve-se levar em consideração que esse valor corresponde a apenas $0,78 \%$ do valor alocado para as ações orçamentárias referentes às despesas correntes e investimentos da Univasf no ano de 2018, e que sua implementação possibilita redução na demanda por pessoal e tempo dedicado a esta atividade.

Por fim, espera-se que esta pesquisa gere desdobramentos, como por exemplo, a decisão pela utilização de etiquetas inteligentes para gestão patrimonial na Univasf, viabilizando a identificação e rastreamento de bens e permitindo a modernização e segurança nos processos envolvidos no controle patrimonial.

\section{SMART TAGS IN THE PUBLIC ADMINISTRATION: FEASIBILITY ANALYSIS IN THE PATRIMONIAL CONTROL OF THE UNIVASF}




\begin{abstract}
The construction of an inventory is not an easy task, especially at federal universities, which have many permanent assets. The patrimonial management is carried through procedures of identification, tipping, localization, counting, preservation and undoing of assets. Due to the large amount of information and the need for security, reliability and speed in the processes, alternatives should be sought to improve management, reduce nonconformity rates, and improve procurement planning. The RFID (Radio Frequency Identification) system, for example, works by means of radio frequency communication, using a passive chip inserted in a smart tag. Thus, the objective of this paper is to analyze the feasibility of using RFID technology to improve asset management at the Federal University of Vale do São Francisco Univasf by surveying its advantages, disadvantages and costs for implementation. For this, research is carried out in journals and patent bases, as well as interviews and document analysis. Among the main results, it can be seen that Univasf's asset management process is carried out manually and exhausting for commission member servers, to which they are summoned without prior consultation, and it is also verified that the deadlines are commonly outdated. On the other hand, the cost of implementing the RFID system to control Univasf's current number of assets corresponds to only $0.78 \%$ of the amount allocated to budget actions related to current expenses and investments, constituting favorable factors for their acquisition by the Institution.
\end{abstract}

Keywords: Radiofrequency. Patrimonial Management. Public universities. Internet of things.

\title{
REFERÊNCIAS
}

ACORDO DE ESTRASBURGO. 24 mar 1971. Disponível em: http://www.inpi.gov.br/menuservicos/informacao/classificacao-patentes. Acesso em: 16 out. 2018.

ALBIERO, Helton Jaques; SILVA, Marcelo Rodrigues da. Economicidade, eficiência e transparência nas compras públicas via pregão eletrônico: estudo de caso dos pregões eletrônicos 42/2012 e 32/2013 realizados pelo IFPR - Campus Assis Chateaubriand. ForScience: revista científica do IFMG, Formiga, v. 6, n. 1, e00275, jan./jun. 2018.

ANDRADE, Menezes Ailson. (ailson.andrade@univasf.edu.br). Relatório Comissão de Inventário de Bens Patrimoniais-2015. [mensagem pessoal]. Mensagem recebida por (welson.santos@univasf.edu.br)em 25 set.2018.

BERNARDES, José Francisco. Gestão Patrimonial: Materiais Permanentes e Bens Móveis. Florianópolis: Imprensa Universitária, 2009.

BERTAGLIA; Paulo Roberto. Tecnologia da informação aplicada à logística. Revista Mundo Logística, v. 34, p. 31, mar./abr. 2013.

BRASIL. Lei n. 4.320, de 17 de março de 1964. Estatui normas gerais de Direito Financeiro para elaboração e controle dos orçamentos e balanços da União, dos Estados, dos Municípios e do Distrito Federal. Brasília: Casa Civil, Subchefia para Assuntos Jurídicos, 1964. Disponível em: http://www.planalto.gov.br/ccivil_03/leis/14320.htm. Acesso em: 17 out. 2018. 
BRASIL. Secretaria de Administração Pública da Presidência da República - SEDAP/PR. Instrução Normativa $\mathbf{n}^{\mathbf{0}} \mathbf{2 0 5}$, de 08 de Abril de 1988. Objetiva racionalização e minimização de custos do uso de material no âmbito do SISG através de técnicas modernas que atualizam e enriquecem essa gestão com as desejáveis condições de operacionalidade, no emprego do material nas diversas atividades. Disponível em:

http://www.comprasnet.gov.br/legislacao/in/in205_88.htm. Acesso em: 17 out. 2018.

COSTA, Alexsander Muniz da. RFControl: sistema de gerência de estoque utilizando RFID. 2018. 48 f. Monografia (Graduação em Engenharia de Computação) - Instituto de Ciências Exatas e Aplicadas, Universidade Federal de Ouro Preto, João Monlevade, 2018. Disponível em: http://www.monografias.ufop.br/handle/35400000/821. Acesso em: 19 jun. 2019.

GIL, Antônio Carlos. Métodos e técnicas de pesquisa social. São Paulo: Atlas, 2008.

HODGES, Steve; MCFARLANE, Duncan. Radio frequency identification: Technology, applications and impact. Cambridge: Cambridge University UK, Auto-ID Lab, 2005.

IDTECHEX. RFID progress at Wal Mart. Apr. 2, 2005. Disponível em: $<$ http://www.idtechex.com/products/en/articles/00000161.asp>. Acesso em: 19 jul. 2019.

INSTITUTO NACIONAL DA PROPRIEDADE INDUSTRIAL (INPI). Disponível em: https://gru.inpi.gov.br/pePI/jsp/patentes/PatenteSearchBasico.jsp. Acesso em: 19 jun. 2019.

LANDT, J. The history of RFID. IEEE Potentials, v. 24, n. 4, p. 8-11, 2005.

MOROZ, R. Understanding Radio Frequency Identification (RFID). Understanding Radio, 2004. Disponível em: http://www.rfidcanada.com/rfid.html. Acesso em: 27 jul. 2019

MOTA, Rafael Perazzo Barbosa. RFID - Radio Frequency Identification. 2012. Monografia (Trabalho acadêmico da disciplina Computação Móvel) - Programa de PósGraduação em Ciência da Computação, Instituto de Matemática e Estatística, Universidade de São Paulo, São Paulo, 2012.

NARCISO, Marcelo Gonçalves. Aplicação da tecnologia de identificação por rádio frequência (Rfid) para controle de bens patrimoniais pela web. Global Science and Technology, v. 1, n. 1, 2008.

ODA, Glauco. O que é RFID: etiquetas RFID no controle patrimonial. AfixCode Patrimônio e Avaliações. São Paulo, 2014. Disponível em: http://www.afixcode.com.br/o-que-e-rfidetiquetas-rfid-controle-patrimonial/. Acesso em: 17 out. 2018.

PREDIGER, D.; SILVEIRA, S. R.; FREITAS, E. P. Modelo de aplicabilidade de sistema RFID para rastreabilidade na indústria alimentícia. Revista de Sistemas e Computação RSC, Salvador, v. 6, n. 1, p. 3-14, jan./jun. Disponível em:

http://www.revistas.unifacs.br/index.php/rsc/article/view/3620. Acesso em: jul. 2019.

GLOSSÁRIO. RFID Journal Brasil, 2019. Disponível em:

http://brasil.rfidjournal.com/glossario. Acesso em: 17 out. 2018. 
RODRIGUEZ, L.; HUERTA, M.; ALVIZU, R.; CLOTET, R. Overview of RFID technology in Latin America. Proceedings of the 6th Andean Region International Conference, Andescon 2012, p. 109-112, 2012.

ROUSSOS, George. Enabling RFID in retail. Computer, v. 39, n. 3, p. 25-30, 2006.

SANGHERA, Paul. RFID: study guide and practice exam. Massachusetts: Syngress Publishing, 2007.

SANTINI, A. G. RFID: conceitos, aplicabilidades e impactos. Rio de Janeiro: Ciência Moderna, 2008.

SILVA, Bruno Cesar. (bruno.cezar@univasf.edu.br). Inventário patrimonial exercício 2018: nova metodologia de trabalho [mensagem pessoal]. Mensagem recebida por (catia.valeria@univasf.edu.br)em25 set.2018.

\section{UNIVERSIDADE FEDERAL DO VALE DO SÃO FRANCISCO - UNIVASF. Plano de Desenvolvimento Institucional-PDI-2016-2025. Petrolina, 2016.}

UNIVERSIDADE FEDERAL DO VALE DO SÃO FRANCISCO - UNIVASF. Pró-Reitoria de Planejamento e Desenvolvimento Institucional. Ato Normativo 001/2018 - PROPLADI: inventário anual - exercício 2018: orientações e procedimentos. Petrolina, 2018. Disponível em: http://portais.univasf.edu.br/propladi/documentos-da-propladi. Acesso em: 18 out. 2018.

USTUNDAG, A.; TANYAS, M. The impacts of Radio Frequency Identification (RFID) technology on supply chain costs. Transportation Research Part E: Logistics and Transportation Review, v. 45, n. 1, p. 29-38, 2009.

YIN, Robert K. Estudo de caso: planejamento e métodos. Porto Alegre: Bookman, 2015.

\section{DADOS DOS AUTORES}

\section{Cátia Valéria dos Santos Passos Brito}

Currículo Lattes: http://lattes.cnpq.br/6816816685472781

E-mail: catia.valeria@univasf.edu.br

Mestranda em Propriedade Intelectual e Transferência de Tecnologia para a Inovação (2018), Universidade Federal do Vale do São Francisco (UNIVASF) Especialista em Educação, Política e Meio Ambiente pelo Instituto Superior de Teologia Aplicada (INTA) (2009) Graduada em Ciências Biológicas pela Universidade de Pernambuco (UPE) (1995). É Servidora, Assistente em Administração do quadro efetivo da Universidade Federal do Vale São Francisco - Univasf, lotada no Núcleo de Inovação Tecnológica - NIT/PRPPGI. Atuou na iniciativa privada, como secretária executiva em algumas empresas (Juazeiro, Salvador, Petrolina, Araripina), não só como facilitadora da vida do executivo, como também participando dos processos internos, obtendo uma visão sistêmica dos processos, missão, objetivos e planejamento estratégico contribuindo com os resultados e com a qualidade do serviço. 


\section{Welson Barbosa dos Santos}

Currículo Lattes: http://lattes.cnpq.br/9186229864419601

E-mail: welson.santos@univasf.edu.br

Mestrando em Propriedade Intelectual e Transferência de Tecnologia para a Inovação (2018) pela UNIVASF. Possui graduação em Ciência da Computação pela Faculdade de Ciências Aplicadas e Sociais de Petrolina (2004). Atualmente é analista de tecnologia da informação da Fundação Universidade Federal do Vale do São Francisco. Tem experiência na área de Desenvolvimento de Sistemas e Redes de Computadores.

\section{Cristiane Xavier Galhardo}

Currículo Lattes: http://lattes.cnpq.br/4119932201644309

E-mail: cristiane.galhardo@univasf.edu.br

Doutorado em Ciências na área de concentração de Química Analítica (2001) pela Universidade de São Paulo (USP) e pós-doutorado (2003) em Química Analítica pelo Centro de Energia Nuclear na Agricultura, CENA, USP, Piracicaba. Possui graduação em Bacharelado em Química Tecnológica pela Universidade Estadual de Campinas (1995), graduação em Licenciatura em Química pela Universidade Estadual de Campinas (1995). Professora Adjunta IV no Colegiado de Engenharia Agronômica da Universidade Federal do Vale do São Francisco, UNIVASF. Tem experiência na área de Química Analítica, com ênfase em Métodos Óticos de Análise, com atenção a técnica espectrofotométrica e quimiluminescência associada a sistemas de Análise por Injeção em Fluxo (FIA), Análise por Injeção Sequencial (SIA) e Multicomutação e Amostragem Binária. Atualmente é docente do Programa de Pós-graduação em Propriedade Intelectual e Transferência de Tecnologia para a Inovação, PROFNIT.

\section{Vivianni Marques Leite dos Santos}

Currículo Lattes: http://lattes.cnpq.br/3040638073236492

E-mail: vivianni.santos@univasf.edu.br

Doutorado em Química pela Universidade Federal de Pernambuco (2004). ProfessoraAssociado IV na UNIVASF. Graduação em Engenharia Química (1997), mestrado em Engenharia Química (2000), na área de Processos Químicos Industriais. Atualmente Diretora Institucional de Inovação Tecnológica. Coordenadora da Regional FORTEC-NE 2019-2020. Coordenadora do Programa de Pós-Graduação em Propriedade Intelectual e Transferência de Tecnologia para Inovação no ponto focal UNIVASF. Docente Permanente no Programa de Pós-Graduação em nível de Doutorado em Agroecologia e Desenvolvimento Territorial. Experiência e formação complementar em Propriedade Intelectual no Japão. Presidente das Comissões Avaliadoras de Pedidos de Patente e de Pedidos de Registros de Programa de Computador sob titularidade da UNIVASF. Docente efetiva do Mestrado Profissional em Gestão Pública no ano de 2017. Editora chefe da Revista Indicação Geográfica e Inovação (INGI). Docente colaboradora no Programa de Pós-Graduação em Engenharia Agrícola da UNIVASF de 2012 a 2017. Coordenadora do Colegiado Acadêmico do Curso de Engenharia de Produção no biênio 11/2009-10/2011 e da CPA/UNIVASF no período de 11/2011 a 03/2016. Líder do grupo de pesquisa: "Propriedade Intelectual e Inovação em Processos, Produtos e Serviços, desde 2009". Experiência nas áreas: Gestão Ambiental, Gestão da Inovação, Agroenergia, Reciclagem, Impactos Ambientais e Modelagem Molecular. Também experiência como docente pesquisadora, orientadora e coordenadora em cursos na modalidade EaD. 